\title{
Ten-Year Clinical Experience with the Lumenless, Catheter-Delivered, 4.1-Fr Diameter Pacing Lead in Patients with and without Congenital Heart
}

\author{
NEHA BANSAL, M.D., SHARMEEN SAMUEL, M.D., KATHLEEN ZELIN, M.S.N., \\ and PETER P. KARPAWICH, M.SC., M.D. \\ From the Section of Pediatric Cardiology, Department of Pediatrics, The Children's Hospital of Michigan, Wayne \\ State University School of Medicine, Detroit, Michigan
}

Background: Patients with congenital heart defects (CHD) often present more challenges to pacing therapy due to anatomy than those without CHD. The lumenless, 4.1Fr diameter M3830 pacing lead (Medtronic, Inc., Minneapolis, MN, USA), approved for use in 2005, has, to date, reported to have excellent short-term ( $<6$ years) lead performance. Unfortunately, very long-term performance is unknown, especially among CHD patients and with implants at alternate pacing (AP) sites. This study reports a 10-year clinical experience with the M3830 lead.

Methods: Records of patients who received the M3830 lead were reviewed: patient demographics, implant techniques and locations, sensing and pacing characteristics, impedances (Imp), and any complications at implant and follow-up.

Results: From 2005 to 2015, 141 patients (ages 2-50, mean 20.1 years, 57\% males) received 212 leads: atrial 115; ventricle 97. CHD was present in $62 \%$ of patients. Leads were inserted at AP sites in $96 \%$ of patients. Postimplant follow-up was from 3 months to 10 years (mean 56.3 months). Comparative implant versus follow-up values (mean \pm standard deviation) were available on 196 leads (92.5\%), showing persistently low $(<1 \mathrm{v} @ 0.4-0.5 \mathrm{~ms})$ pacing thresholds $(P=0.57)$. Sensing was also comparable (atrial leads, $P=0.41$; ventricular leads, $P=0.9)$. Impedances differed $(P<0.05)$ but remained within the normal range. Two $A$ leads became dislodged and one was repositioned while two other leads $(1 \mathrm{~A}, 1 \mathrm{~V})$ were extracted. There are no differences observed in the pacing characteristics between the CHD and non-CHD groups on follow-up.

Conclusions: The 4.1Fr lumenless pacing lead shows ease of implant regardless of CHD or AP site, excellent very long-term (10 years) stability, and performance indices with a very low rate of complications. (PACE 2017; 40:17-25)

congenital heart defect, pacing, pediatrics, lumenless pacing lead

\section{Introduction}

The management of congenital heart disease (CHD) patients continues to be challenging even after surgical repair, which, due to conduction system damage, often results in the need for lifelong pacing therapy. Due to the persistence of altered anatomy and vascular issues, pacing lead implantation is associated with more technically demanding issues than among non-CHD patients. ${ }^{1}$ These issues are often compounded by small

Conflict of Interests: None declared.

Address for reprints: Peter P. Karpawich, M.Sc., M.D., Director, Cardiac Electrophysiology, The Children's Hospital of Michigan, 3901 Beaubien Blvd, Detroit, MI 48201. Fax: 313993-0894; e-mail: pkarpawi@dmc.org

Received April 19, 2016; revised October 27, 2016; accepted November 26, 2016.

doi: 10.1111/pace.12995 patient body sizes and vascular dimensions, creating a high risk for venous obstruction, reportedly ranging from $13 \%$ to $64 \%$ of patients. ${ }^{2-5}$ With the advances in technology, new pacing lead designs have evolved over time, offering benefits among challenging patient groups such as those with CHD. Such new designs have improved implantation/extraction at alternative and often technically difficult sites to access, while still assuring limited complications (e.g., dislodgements, fractures, and venous occlusions), as well as causing adverse effects on valves and vasculature. ${ }^{6}$

One of the newest designs is the lumenless, 4.1Fr diameter, steroid-eluting, coaxial, solid core, nonstylet, catheter-delivered lead (M3830, Medtronic, Inc., Minneapolis, MN, USA). Approved for use in the USA in 2005, the flexible catheter-delivery technique permits ease of implant at traditional (appendage/apex) as well as nonapical and appendage locations. These

(C2016 Wiley Periodicals, Inc. 
latter locations can improve paced myocardial function. ${ }^{7}$ Although relatively short-term performances ( $<6$ years, mean 2 years) have been published, very long-term efficacy of this lead design has not been reported, especially among CHD patients and with implant at alternate (nonappendage/apex) pacing (AP) sites. ${ }^{8,9}$

This study is a retrospective review of chronic M3830 lead performance indices at a single congenital heart center from 2005 to 2015. It presents the longest postimplant (up to 10 years postimplant, mean 56.3 months) evaluation of this lead's pacing and sensing performances, in all age patients, and any complications as well as extraction concerns among patients with normal anatomy as well as various pre- and postrepair congenital heart defects.

\section{Materials}

The Medtronic M3830 lead is a lumenless active fixation, bipolar, steroid-eluting (initially declomethasone, changed to beclomethasone after 2012) helical design lead. The 4.1-Fr isodiametric lead body has a solid core with an exposed helix. Removal of the central lumen, necessary for styletdelivered leads, effectively reduces the diameter up to $40 \%$ of a standard 5-7-Fr pacing lead, permitting transvenous pacing among younger patients, especially those with repaired or unrepaired congenital heart defects. ${ }^{10}$ The M3830 lead employs a central cable conductor coil with silicone inner and polyurethane outer insulations, available in 49-59-69-cm lengths. The use of composite insulation material (polyurethane plus silicone) not only contributes in diameter reduction, but also results in improved crush resistance and ease of insertion through the accompanying delivery catheter, an $8.4 \mathrm{Fr}$ diameter steerable slice-away sheath (SelectSecure ${ }^{\mathrm{TM}}$ ), which has kink-resistant properties. The flexibility of this kink-resistant catheter permits from 22 to $44 \mathrm{~cm}$ long curves, which facilitate implant at select and alternate sites, including septal, left atrial, and outflow regions, which greatly facilitate implant in some more complex CHD anatomies (Fig. 1). In addition, use of smaller diameter introducer sheaths or direct insertion without use of any sheaths as well as a transatrial approach have been successfully reported..$^{1,11,12}$

\section{Methods}

This study is a retrospective review of the M3830 lead performance indices (sensing and pacing thresholds, impedances), as well as any technical implant or explant issues, from January 2005 to December 2015. The institutional review board at The Children's Hospital of Michigan/Wayne State University

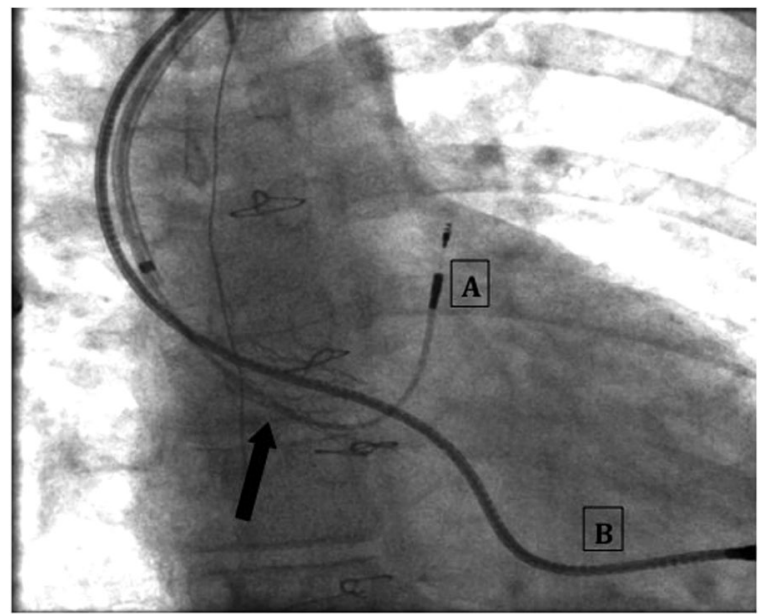

Figure 1. Fluoroscopic (AP view) comparative appearance of the 4.1Fr M3830 lead (A) and more standard $6 F r$ lead (B) implanted in the venous left atrium and ventricle in a patient following the Mustard atrial baffle repair for D-TGA. The smaller diameter of the M3830 lead is readily apparent. Lead implant at the roof of the left atrium, and avoidance of phrenic nerve stimulation, was easily achieved by use of the flexible delivery catheter which is able to overcome the acute angle often caused by an intravascular stent placed in the superior Mustard baffle (arrow).

School of Medicine approved the study. Inpatient and outpatient charts and electronic records were reviewed. Patient demographics, congenital cardiac anatomies, surgical repairs, lead implant sites, lead sensing, and pacing properties at implant and follow-up clinic visits were collected. Complications at the time of implantation as well as during follow-up requiring lead extraction/revision were also recorded. All patients had a predevice implant echocardiography/Doppler study.

Implant technique: As previously described, following a standard sterile surgical pacemaker implantation protocol, either the left or right subclavicular approach, was used. ${ }^{9}$ Initially, a standard right and left heart catheterization study was performed on all patients from the femoral approach to include evaluation of vascular patency and access routes to ascertain any leaks/shunting that might require closure or vessel/baffle narrowing that might require stent placement prior to lead implant. For all patients, optimal lead pacing and sensing characteristics were achieved. Physiologic optimization of paced-contractility at all sites was undertaken by comparative online measurements of the systemic ventricle's contractility indices 
(dP/dt-max) at various potential implant sites along the ventricular septum for ventricular pacing, and at the atrial-free wall or septum for atrial pacing. Two-dimensional, color-guided spectral Doppler, and tissue Doppler imaging of left atrial ejection fraction determined the choice of atrial implant sites. ${ }^{13}$ The subclavian vein was entered using the standard needle approach. Most implants utilized the SelectSecure ${ }^{\mathrm{TM}}$ catheter delivery system. Other implant variations included use of smaller diameter introducers depending on patient size and anatomy as well as the transatrial approach to ventricular lead implant. ${ }^{12}$ All Model 3830 leads were positioned without difficulty. Standard measurements of sensing and pacing indices were recorded in each instance. Since this was a new lead design and delivery system when first introduced, fluoroscopy times for lead implant were initially recorded. ${ }^{9}$ Following implanter familiarity with this lead, however, continued recording of specific implant times was not routinely performed. All single ventricle patients were routinely treated with aspirin/coumadin depending on any recurrent clinical flutter. Postdevice implant, anticoagulation therapies were not modified.

Patients were followed in the electrophysiology clinic at the Children's Hospital of Michigan section of Cardiology at regular intervals per protocol. Pacemaker checks were performed at these visits to record each lead's performance indices. These records were kept in the pacemaker charts as well as recorded electronically in the patients' electronic records.

Statistics: Data were analyzed using the GraphPad software QuickCalcs using exact Fisher's test and paired $t$-test with P-value $<0.05$ considered statistically significant.

\section{Results}

Performance data on all 212 leads (115 atrial; 97 ventricular) from 141 patients was reviewed. Patients (57\% males) ranged in age from 2 to 50 years (mean 20.1 years). Both pre- $(27 \%)$ and postrepair $(73 \%$ of patients) CHD included septal defects, tetralogy of Fallot, and transposition of the great arteries [TGA; both dextro (D) and levo (L)] (Table I). Leads were implanted at AP sites in $96 \%$ of patients including, but not restricted to, the right atrial septum, ventricular septum, and left atrium/left ventricle (D-TGA with the Mustard intra-atrial baffle repair). Among patients with single ventricle anatomy, both the classic atrio-pulmonary and lateral tunnel surgical "Fontan" procedures had been performed. Among those with the lateral tunnel repair, effective transvenous atrial pacing

\section{Table I.}

Patient Implant Demographics

\begin{tabular}{lc}
\hline Total leads & 212 \\
Total patients & 141 \\
Atrial leads & 115 \\
Ventricular leads & 97 \\
Implant age in years (mean) & $2-50(20.1)$ \\
Male: female & $81: 60(57.5 \%: 42.5 \%)$ \\
Normal cardiac anatomy & $53 / 141(38 \%)$ \\
CAVB & $33 / 53(62 \%)$ \\
Cardiomyopathy & $9 / 53(17 \%)$ \\
Long QTC & $7 / 53(13 \%)$ \\
Ventricular tachycardia & $4 / 53(8 \%)$ \\
Structural CHD & $\mathbf{8 8 / 1 4 1}(62 \%)$ \\
D-TGA & $30 / 88(34 \%)$ \\
TOF & $14 / 88(16 \%)$ \\
Single ventricle physiology & $11 / 88(12.5 \%)$ \\
VSD & $8 / 88(9 \%)$ \\
AS/aortic or mitral valve & $8 / 88(9 \%)$ \\
$\quad$ replacement & \\
AV canal & $7 / 88(8 \%)$ \\
L-TGA & $5 / 88(5.7 \%)$ \\
ASD & $2 / 88(2.3 \%)$ \\
Others & $3 / 88(3.5 \%)$ \\
\hline AS
\end{tabular}

$\mathrm{AS}=$ aortic stenosis; $\mathrm{ASD}=$ atrial septal defect; $\mathrm{AV}=$ atrioventricular; CAVB = complete atrioventricular block; CHD = congenital heart defects; D-TGA = dextro-transposition of the great arteries; L-TGA = levo-transposition of the great arteries; TOF $=$ tetralogy of Fallot; VSD = ventricular septal defect.

was achieved by lead insertion at the atrial remnant along the cavopulmonary anastomosis ${ }^{14}$ (Fig. 2). One patient with complex CHD (hypoplastic right ventricle, bilateral "Glenn" pulmonary artery connections, and atrial septal defect) with fractured epicardial leads, underwent a transatrial ventricular implant approach (Fig. 3).

All leads were implanted without complications with standard follow-up clinical evaluations. More than $50 \%$ of patients were followed for greater than 5 years postimplant. Data on all 212 leads showed the implant pacing threshold (mean \pm standard deviation) (at $0.4-0.5 \mathrm{~ms}$ ) to be $0.69 \pm 0.32 \mathrm{v}$. Comparative follow-up data at more than 3 months to 10 years (mean 56.3 months) could be obtained on 196/212 leads (92.5\%): 106 atrial and 90 ventricular leads. Patients with the remaining 16 leads were lost to followup ( $<3$ month follow-up) or died soon after implant for nonpacemaker-related issues. There was no evidence of lead failure. Pacing thresholds (measured in volts at $0.4-0.5-\mathrm{ms}$ pulse width) remained persistently low for all the 196 leads for which chronic data were available. When taken 


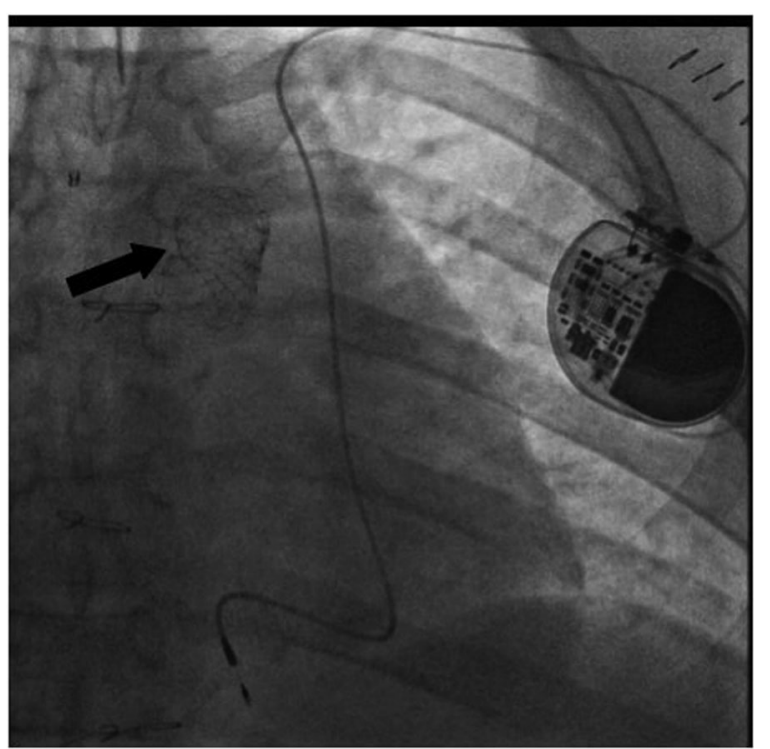

Figure 2. Fluoroscopic (AP) view of a M3830 lead implanted at the atrial remnant site in a patient following the lateral tunnel "Fontan" repair for a single ventricle. There was an absent innominate vein with a left superior vena cava opening into the venous atrium. The lead was easily positioned and secured in a very small area of electrically active residual atrial tissue that permitted effective pacing and sensing. Note a stent (arrow) in the aorta at the site of a previous coarctation.

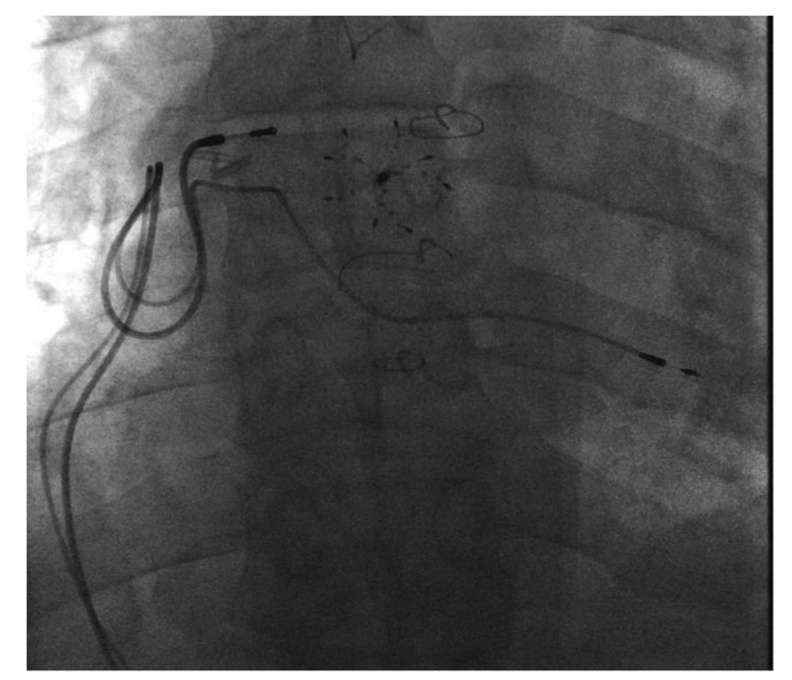

Figure 3. Fluoroscopic (right anterior oblique) view of a M3830 lead placed via a transatrial approach into a hypoplastic right ventricle. A larger $6 \mathrm{Fr}$ diameter lead is secured to the atrium. Note the spider-like Cardioseal ${ }^{\mathrm{TM}}$ atrial septal defect closure device. as a whole, there was no significant difference in thresholds: $0.69 \pm 0.31 \mathrm{v}$ (at implant) versus $0.67 \pm 0.35 \mathrm{v}$ (at last follow-up) ( $\mathrm{P}=0.57$ ). When these data were divided into chamber paced, atrial (A) lead thresholds either remained constant or showed some decrease $(\mathrm{P}<0.05)$ at certain postimplant intervals. In comparison, individual ventricular (V) lead thresholds either remained consistent or showed a specific timeinterval increase $(\mathrm{P}<0.05)$ when compared with implant values, but remained $<1 \mathrm{v}$ (Fig. 4). Both $P$ and $R$ wave sensing remained stable over the duration of the study (Fig. 5). As seen in Table II, when all leads were taken as a whole, comparing implant with the most recent followup data, sensing characteristics were comparable from implant throughout the study: P waves [mean $3.65 \pm 1.95$ vs. $3.63 \pm 2 \mathrm{mv}(\mathrm{P}=0.93)]$ and $\mathrm{R}$ waves $[10.5 \pm 5.5$ vs. $9.9 \pm 4.8 \mathrm{mv}(\mathrm{P}=0.41)]$. When leads were serially compared over years of follow-up, there were changes in impedances [atrial: $745 \pm$ 226 vs. $553 \pm 119 \Omega$ and ventricular: $847 \pm 254$ vs. $521 \pm 80 \Omega(\mathrm{P}<0.0001)]$ but all within the normal range (350-1,500 $\Omega$ ) for this particular structural design. There was no evidence of conductor coil fracture nor insulation breaks.

On comparison of patients who had structural CHD $(62 \%)$ with those who had a structurally normal heart (38\%), no differences were found in the sensing characteristics. $P$ waves [mean $3.46 \pm$ $2 \mathrm{mv}$ vs. $3.82 \pm 2(\mathrm{P}=0.37)]$ and $\mathrm{R}$ waves $[8.7 \pm$ $4.7 \mathrm{mv}$ vs. $10.75 \pm 5.3(\mathrm{P}=0.08)]$. There was also no significant difference in thresholds observed at the last follow-up: atrial leads: $0.61 \pm 0.4 \mathrm{v}$ (nonCHD patients) vs. $0.58 \pm 0.32 \mathrm{v}$ (CHD patients) $(\mathrm{P}=0.65)$ and ventricular leads: $0.77 \pm 0.3 \mathrm{v}$ (nonCHD patients) vs. $0.71 \pm 0.35 \mathrm{v}$ (CHD patients) $(\mathrm{P}=0.45)$. All the impedances were in the normal range and no differences were observed between either the atrial (566.9 \pm 141 vs. $532 \pm 62.8$; $\mathrm{P}=$ 0.13 ) or ventricular leads (525 \pm 76.5 vs. $515.3 \pm$ 86.4; $\mathrm{P}=0.57)$.

Complications, in the form of dislodgement, were minimal (1\%). Two A leads became dislodged $<1$ month postimplant, both associated with excessive patient arm and shoulder activity. Both the leads were implanted in the right atrial septal position. One was abandoned and the other was repositioned using a custom-made tool design. ${ }^{15}$ Two other leads ( $\left.1 \mathrm{~A}, 1 \mathrm{~V}\right)$ were extracted at the time of the initial device exchange to an implantable cardioverter defibrillator, 4 years after the M3830 leads were implanted. Of note, extraction by simple traction of neither lead was possible due to excessive adhesions along the innominate-superior vena cava region. Extraction was then successfully performed by use of an $8 \mathrm{Fr}$ 
A

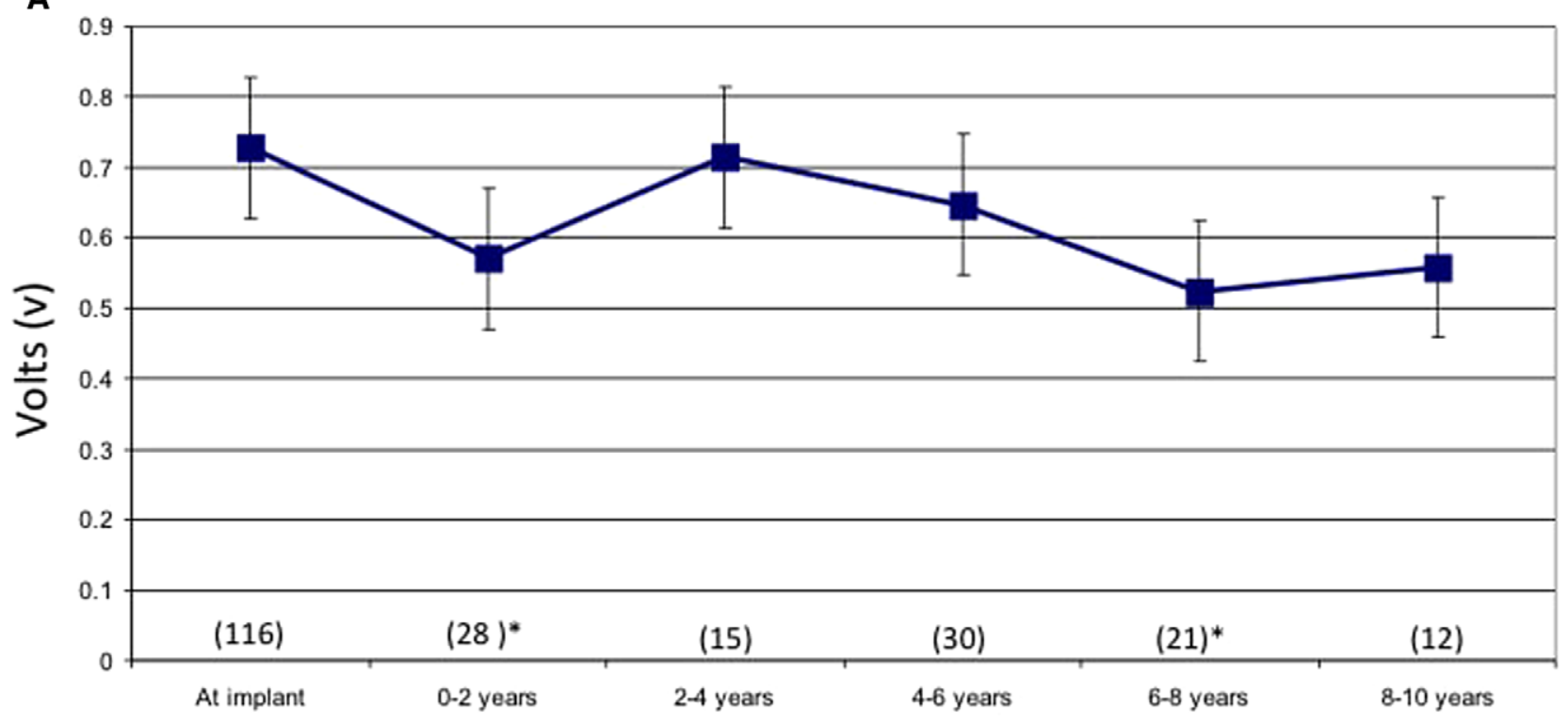

B

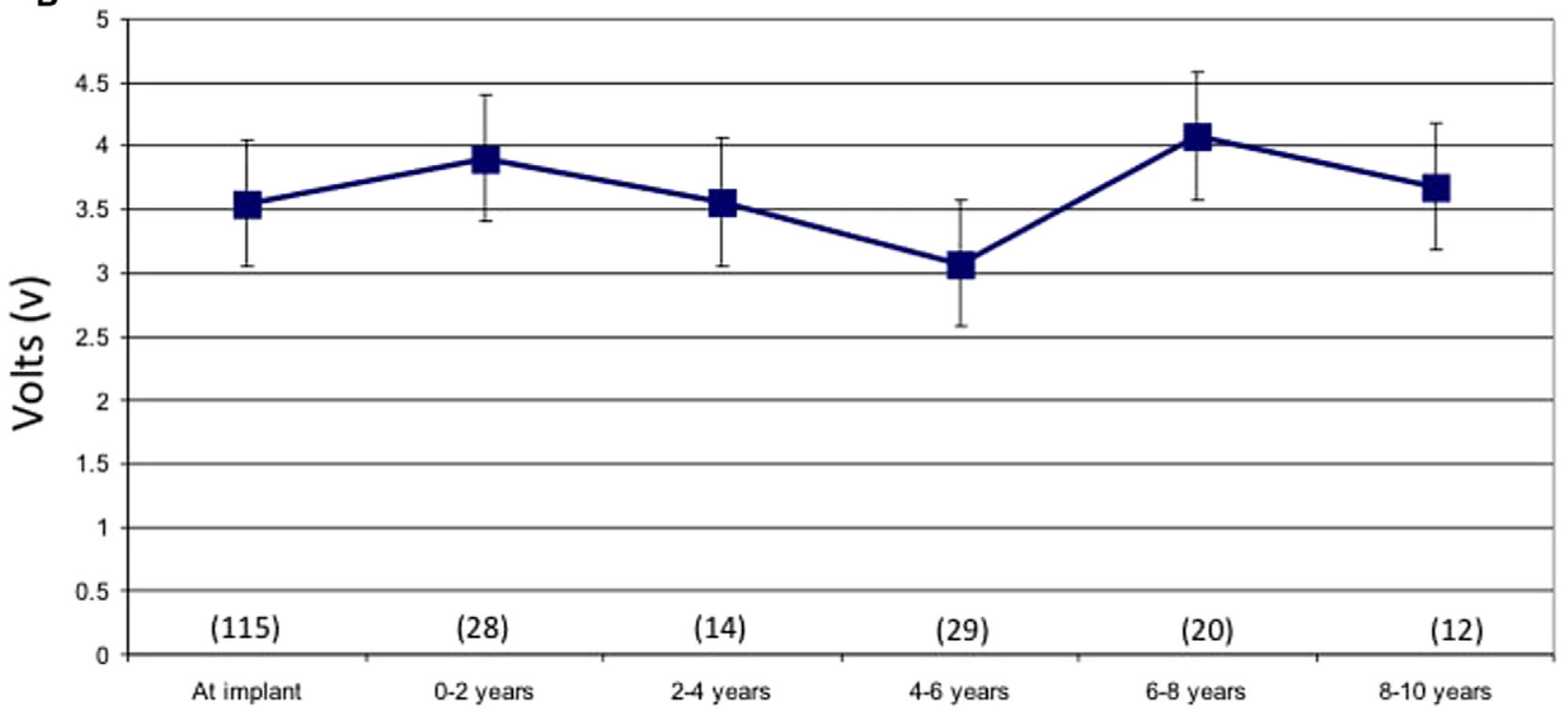

Figure 4. Pacing thresholds. (A) Atrial voltage thresholds (mean $\pm S D$ ) measured at 0.4-0.5 ms, from implant over time with the number of leads for each follow-up period indicated. Lower thresholds $(P<0.05)$ were observed at the $0-2$ years and 6-8 years follow-up intervals (marked by *). Overall mean threshold values were $<1 \mathrm{~V}$. (B) Ventricular voltage thresholds (mean $\pm S D$ ) measured at 0.4-0.5 ms, from implant over time with the number of leads for each follow-up period. Higher mean thresholds $(P<0.05)$ were observed at the $0-2$ years and 4-6 years follow-up (marked by *). Overall, mean threshold values were $<1$ v. $S D=$ standard deviation. [Color figure can be viewed at wileyonlinelibrary.com]

counter traction catheter (Yellowbird, Cook, Inc., Bloomington, IN, USA).

\section{Discussion}

With the technical advances in the pacing lead designs, physicians are moving toward placement of transvenous pacemakers in younger patients and also in CHD patients. ${ }^{16-24}$ However, this unique population poses numerous challenges for stable, long-term pacing success. ${ }^{25}$ These include a smaller patient size and thus smaller vascular calibers, diverse venous anatomies, and 

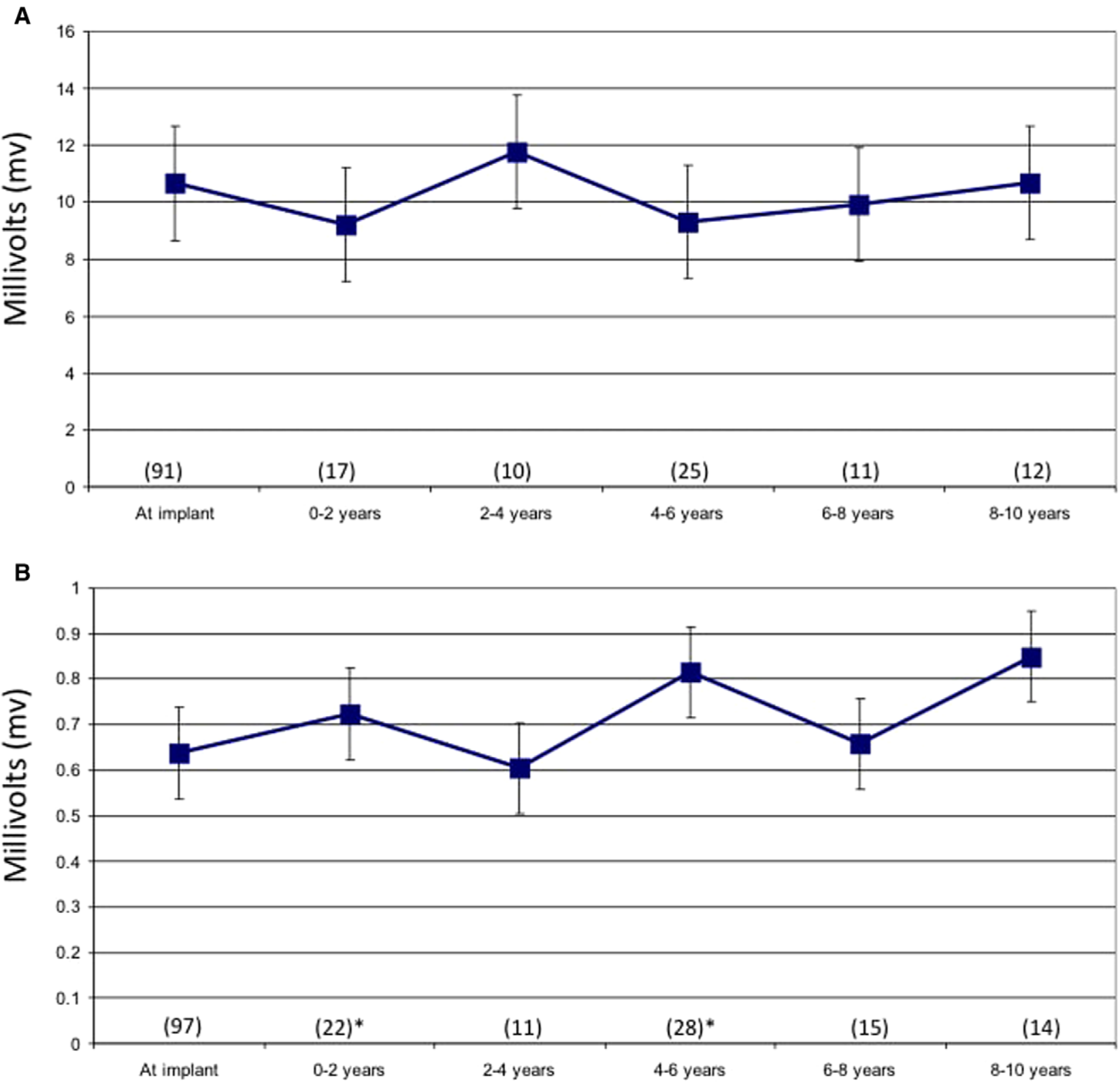

Figure 5. Sensing performances. (A) Atrial $P$ wave (mean $\pm S D$ ) sensing ( $m V$ ) over time with the number of leads for each follow-up period (parenthesis). No significant changes were observed at any specific follow-up period. (B) Ventricular $R$ wave (mean $\pm S D$ ) sensing $(\mathrm{mV})$ over time with the number of leads for each follow-up period (parenthesis). No significant changes were observed at any specific follow-up period. SD = standard deviation. [Color figure can be viewed at wileyonlinelibrary.com]

structural defects and various repairs, which can alter normal anatomy. All of these factors can complicate and even prohibit typical delivery and lead positioning techniques. In addition, somatic growth and active patient lifestyles can affect lead stability. ${ }^{20,23}$ Another challenge to implant is the achievement of select site placement to improve paced myocardial function, with stylet-delivered leads. ${ }^{9,10,26,27}$
The relatively new, lumenless, $4.1 \mathrm{Fr}$ diameter pacing lead (Medtronic Inc.) has gained particular attention in pediatric/congenital heart cardiology centers as it appears to minimize complications typically associated with standard transvenous leads, especially among younger patients, as noted in this study, and to provide potential advantages in CHD and pediatric patient population due to its specific design. This lead is an active 
Table II.

All Leads Performance Indices`@

\begin{tabular}{|c|c|c|c|c|c|c|}
\hline \multirow[b]{2}{*}{ Parameter } & \multicolumn{3}{|c|}{ Atrial Leads $($ mean \pm SD) $(n=115)$} & \multicolumn{3}{|c|}{ Ventricular Leads (mean \pm SD) $(n=97)$} \\
\hline & At Implant & At Follow-Up & P-Value & At Implant & At Follow-Up & P-Value \\
\hline $\begin{array}{l}\text { Pacing threshold } \\
\text { (volts @ } \\
0.4-0.5 \mathrm{~ms} \text { ) }\end{array}$ & $0.73 \pm 0.3$ & $0.6 \pm 0.35$ & 0.0029 & $0.64 \pm 0.32$ & $0.75 \pm 0.34$ & 0.0063 \\
\hline $\begin{array}{l}\text { Sensed P/R wave } \\
\text { (mv) }\end{array}$ & $3.65 \pm 1.95$ & $3.63 \pm 2$ & 0.93 & $10.5 \pm 5.5$ & $9.9 \pm 4.8$ & 0.41 \\
\hline $\begin{array}{l}\text { Lead impedance } \\
\quad(\text { ohms, } \Omega)\end{array}$ & $745 \pm 227$ & $553 \pm 119$ & $<0.0001$ & $847 \pm 254$ & $521 \pm 80$ & $<0.0001$ \\
\hline
\end{tabular}

${ }^{*}$ mean \pm SD; at implant and follow-up values are composites of all leads obtained at implant and most recent postimplant interval. SD $=$ standard deviation.

fixation, bipolar, steroid-eluting (currently lipidsoluable beclomethasone) design with a solid core. It is the thinnest diameter lead currently available. With the growing population of adult patients with CHD, the demand for small diameter pacing leads has increased and, therefore, the lumenless, nonstylet delivered coaxial leads offer improved implant capabilities and crush resistance. ${ }^{10}$

A potential advantage of the lead is the ease of implantation, stability, and electrode securing capabilities at alternate pacing sites. This is especially important among CHD patients. Previous studies have demonstrated that chronic pacing at the more traditional ventricular apical or atrial appendage implant sites can adversely affect myocardial function. ${ }^{7,28}$ Additionally, among CHD patients even with repaired hearts, the traditional implant sites may be difficult if not impossible to attain for effective pacing. ${ }^{29}$ For example, the right atrial appendage is often amputated following bypass cannulation and ventricular-pulmonary conduits may distort the apex. Short-term experience with this M3830 lead in patients with CHD at traditional pacing sites, as well as alternative pacing sites, has been promising. ${ }^{30}$ Device implant among repaired congenital heart patients can be challenging. In the current era, with fewer diagnostic cardiac catheterizations being performed, compared to the pre-2D echocardiography era, catheter manipulation skills may require extra learning. However, once mastered, in our experience, this catheterdelivered lead surpasses the older stylet-delivered leads, especially in attempting to achieve the more patient-beneficial "alternate site" implants which previously often required multiple attempts to form the most appropriate stylet curves. In two previous studies, fluoroscopy times for the M3830 lead implant ranged from 0.45 to 9 minutes (mean $1.6 \pm 1.3$ ) and 1.8 to 90 minutes (mean16.2 \pm 14.7)..$^{8,9}$

In this report, $>95 \%$ of the leads were implanted at alternate pacing sites (i.e., right atrial septum or left atrial-free wall, right or left ventricular septum, and atrial remnants among patients having undergone the lateral-tunnel "Fontan") with excellent pacing/sensing results and minimal complication rates for a postimplant interval of 5 years (follow-up data available for $>50 \%$ of the patients) and 10 years (follow-up data available for $>20 \%$ of the patients). Although "His-" and "para-His-bundle" pacing has been reported with this lead, thresholds are often much higher than at other septal sites, indicating a problem more with the implant site than lead characteristics. ${ }^{31}$ Since the maintenance of low thresholds, and associated low generator outputs which extend battery longevity, is paramount when considering pacemaker therapy in the young, this particular site was purposely not utilized. Another favorable aspect of the use of this lead is its improved clinical benefits on the integrity of atrioventricular valves and venous channels, reducing the rate of valvular insufficiency and venous obstruction when compared to the more traditional and larger diameter leads. ${ }^{6}$

As noted above, although a few studies have been published which report on the short-term performance of the M3830 pacing lead since its approval, the very long-term evaluation is still unknown. This limits the knowledge of chronic efficacy and also any complications that may or may not arise in association with this design, 
placement, and extraction. A recent retrospective study compared the M3830 with a different lead (M1488, St. Jude Medical Inc., St. Paul, MN, USA) over a 6-year (mean 26 months) interval. The report demonstrated similar efficacy of both leads among children and CHD patients but with different complication rates: $5 \%$ among patients who received the M3830 as compared to $20 \%$ among those who received the M1488 lead, as well as easier maneuverability of the M3830. Of note, and different from this current report, nine leads were extracted successfully by use of only manual traction without the need for tools. ${ }^{8}$ However, the average postimplant duration was 1.37 years. Unfortunately, although that study did indicate that at least one extraction was in a patient with a dual-chamber system, time after lead implant was not indicated. Therefore, presence of a dual-chamber system, as seen in our experience, may compound and accelerate any lead-endothelial interactions, negating simple extraction without tools. Further evaluations of vascular effects on the M3830 lead are required before any definitive assessments of lead-vascular interactions and extraction can be made.

Results of this current report are similar to those from a previous study demonstrating the 5-year postimplant performances indices of the M3830 lead compared with more traditional stylet-delivered leads. This latter study focused particularly on the efficacy and stability of the M3830 leads implanted at alternate pacing sites and concluded that performance indices were comparable. ${ }^{9}$ A few smaller studies that were done among CHD patients, with limited followup intervals, also demonstrated impressive performance of the lead with excellent pacing/sensing thresholds and normal impedance values. . $^{26,27,29,32}$ To date, most studies done on the performance evaluation of M3830 leads have demonstrated a low to acceptable rate of complications related to the lead implant, comparable to traditional leads.

However, the information about complications related specifically to M3830 lead is limited. A case report addressed the issue of difficult repositioning after dislodgement of the lead, due to the absence of an internal stylet, which facilitates the lead repositioning. This necessitated fabrication of an innovative tool design. ${ }^{15}$ That, perhaps, is the one potential shortcoming of this lead: lack of ease of repositioning in instances of dislodgement. Fortunately, dislodgement was not a prominent feature during this 10-year followup study. One of the original multicenter studies reported problems related to lead placement at a follow-up interval of 3 months which included elevated pacing thresholds $(0.5 \%)$, failure to sense $(0.5 \%)$, pericardial effusion $(0.6 \%)$, cardiac tamponade $(0.2 \%)$, and lead dislodgement $(2.3 \%)$. Some of these complications were related to the lead placement technique. The most frequently observed complication among previously published reports was lead dislodgement. ${ }^{10,30}$ In this study, only two of the 115 atrial leads (1\%) and no ventricular leads became dislodged. Implant technique may be a definitive factor with implant experience contributing to more lead stability. The observed differences in lead impedances seen in this study are typical of most chronic lead studies and reflect more on intrinsic characteristics of electrical conductance, not any lead problem, per se. Likewise, changes in voltage thresholds, although different over time, and associated patient growth remained well within acceptable values of $\leq 1 \mathrm{v}$.

\section{Conclusion}

With the improved care and management of patients with congenital heart defects, there has been an increase in the adult population of patients with CHD, thus increasing the demand of more maneuverable pacing leads that permit fixation at sites other than right ventricular apical or atrial appendage locations. Thinner diameter leads with improved stability add additional benefits of less adverse effects on valves and vasculature, which are important in the young. The novel 4.1-Fr diameter lumenless pacing lead is unique in its design compared with more traditional lead designs (5-7Fr), permitting transvenous pacing in young patients with or without CHD with limited adverse valve and vascular problems. The provided catheter-based delivery of the lead has resulted in improved maneuverability and low complication rates when compared to the more conventional leads. In addition, the M3830 lead can be inserted with none or smaller diameter catheters. In this study, the $4.1 \mathrm{Fr}$ diameter, lumenless pacing lead exhibited ease of implant regardless of CHD or site, excellent very long-term (up to 10 years) stability, and performance indices with a very low rate of complications.

\section{Study Limitations}

This is a retrospective study that analyzed pacing lead data available for patients followed at a single tertiary cardiac center with implant by established physicians who were very familiar with the lead's characteristics. Further multicenter studies with implant by various physicians of different skill sets are needed, possibly with comparisons with various other leads, to further assess the efficacy of this pacing lead in more diverse patient populations and clinical settings. 
Nevertheless, the M3830 lead design shows exceptional long-term performance in the very

\section{References}

1. Fishberger SB, Rollinson NR, Warsy I, Whang B, Kim RW. Transatrial lead implantation using the 4-Fr lumenless pacing lead and delivery system in young adults with congenital heart disease. Pacing Clin Electrophysiol 2009; 32:e40-2.

2. Bar-Cohen Y, Berul CI, Alexander ME, Fortescue EB, Walsh EP, Triedman JK, Cecchin F. Age, size, and lead factors alone do not predict venous obstruction in children and young adults with transvenous lead systems. J Cardiovasc Electrophysiol 2006; 17:754-759.

3. Bracke F, Meijer A, Van Gelder B. Venous occlusion of the access vein in patients referred for lead extraction: Influence of patient and lead characteristics. Pacing Clin Electrophysiol 2003; 26:16491652.

4. Da Costa SS, Scalabrini Neto A, Costa R, Caldas JG, Martinelli Filho $\mathrm{M}$. Incidence and risk factors of upper extremity deep vein lesions after permanent transvenous pacemaker implant: A 6-month followup prospective study. Pacing Clin Electrophysiol 2002; 25:13011306.

5. Figa FH, McCrindle BW, Bigras JL, Hamilton RM, Gow RM. Risk factors for venous obstruction in children with transvenous pacing leads. Pacing Clin Electrophysiol 1997; 20:1902-1909.

6. Bharmanee A, Zelin K, Sanil Y, Gupta P, Karpawich PP. Comparative chronic valve and venous effects of lumenless versus stylet-delivered pacing leads in patients with and without congenital heart. Pacing Clin Electrophysiol 2015; 38:1343-1350.

7. Giudici MC, Karpawich PP. Alternative site pacing: it's time to define terms. Pacing Clin Electrophysiol 1999; 22:551-553.

8. Garnreiter J, Whitaker P, Pilcher T, Etheridge S, Saarel E. Lumenless pacing leads: Performance and extraction in pediatrics and congenital heart disease. Pacing Clin Electrophysiol 2015; 38:42-47.

9. Khan A, Zelin K, Karpawich PP. Performance of the lumenless 4.1-Fr diameter pacing lead implanted at alternative pacing sites in congenital heart: A chronic 5-year comparison. Pacing Clin Electrophysiol 2010; 33:1467-1474.

10. Gammage MD, Lieberman RA, Yee R, Manolis AS, Compton SJ, Khazen C, Schaaf K, et al. Multi-center clinical experience with a lumenless, catheter-delivered, bipolar, permanent pacemaker lead: Implant safety and electrical performance. Pacing Clin Electrophysiol 2006; 29:858-865.

11. Kenny D, Walsh KP. Noncatheter-based delivery of a singlechamber lumenless pacing lead in small children. Pacing Clin Electrophysiol 2007; 30:834-838.

12. Lapage MJ, Rhee EK. Alternative delivery of a $4 \mathrm{Fr}$ lumenless pacing lead in children. Pacing Clin Electrophysiol 2008; 31: 543-547.

13. Zilberman MV, Karpawich PP. Alternate site atrial pacing in the young: Conventional echocardiography and tissue Doppler analysis of the effects on atrial function and ventricular filling. Pacing Clin Electrophysiol 2007; 30:755-760.

14. Rosenthal E, Konta L. Transvenous atrial pacing from the superior vena cava stump after the hemi-fontan operation-A new approach. Pacing Clin Electrophysiol 2014; 37:531-536.

15. Karpawich PP, Webster P, Goodman E, Forbes T. Repositioning a dislodged new lumenless pacing lead: A simple tool and technique. Pacing Clin Electrophysiol 2008; 31:354-357. complex and diverse as well as aging congenital heart patient population.
16. Ayabakan C, Rosenthal E. Endocardial pacemaker implantation in neonates and infants. Indian Pacing Electrophysiol J 2006; 6:57-62.

17. Kerstjens-Frederikse MW, Bink-Boelkens MT, de Jongste MJ, Homan van der Heide JN. Permanent cardiac pacing in children Morbidity and efficacy of follow-up. Intl J Cardiol 1991; 33:207214.

18. Nordlander R, Pehrsson SK, Book K, Zetterqvist P, Fahlen-Vepsa IL. Clinical experience of pacemaker treatment in children. Scand J Thoracic Cardiovasc Surg 1992; 26:69-72.

19. Robledo-Nolasco R, Ortiz-Avalos M, Rodriguez-Diez G, JimenezCarrillo C, Ramirez-Machuca J, De Haro S, Castro-Villacorta H. Transvenous pacing in children weighing less than 10 kilograms. Pacing Clin Electrophysiol 2009; 32:S177-S181.

20. Sachweh JS, Vazquez-Jimenez JF, Schondube FA, Daebritz SH, Dorge H, Muhler EG, Messmer BJ. Twenty years experience with pediatric pacing: Epicardial and transvenous stimulation. Eur J Cardio-Thoracic Surg 2000; 17:455-461.

21. Silvetti MS, Drago F, Grutter G, De Santis A, Di Ciommo V, Rava L. Twenty years of paediatric cardiac pacing: 515 pacemakers and 480 leads implanted in 292 patients. Europace 2006; 8:530-536.

22. Till JA, Jones S, Rowland E, Shinebourne EA, Ward DE. Endocardial pacing in infants and children $15 \mathrm{~kg}$ or less in weight: Medium-term follow-up. Pacing Clin Electrophysiol 1990; 13:1385-1392.

23. Walsh CA, McAlister HF, Andrews CA, Steeg CN, Eisenberg R, Furman S. Pacemaker implantation in children: A 21-year experience. Pacing Clin Electrophysiol 1988; 11:1940-1944.

24. Ward DE, Jones S, Shinebourne EA. Long-term transvenous pacing in children weighing ten kilograms or less. Intl J Cardiol 1987; $15: 112-115$

25. Silka MJ, Bar-Cohen Y. Pacemakers and implantable cardioverterdefibrillators in pediatric patients. Heart Rhythm 2006; 3:13601366.

26. Cantu F, De Filippo P, Gabbarini F, Borghi A, Brambilla R, Ferrero P, Comisso J, et al. Selective-site pacing in paediatric patients: A new application of the Select Secure system. Europace 2009; 11:601606.

27. Tuzcu V. Implantation of SelectSecure leads in children. Pacing Clin Electrophysiol 2007; 30:831-833.

28. Prinzen FW, Augustijn CH, Arts T, Allessie MA, Reneman RS. Redistribution of myocardial fiber strain and blood flow by asynchronous activation. Am J Physiol 1990; 259:H300-H308.

29. Mond H, Karpawich PP. Pacing Options in the Adult Patients with Congenital Heart Disease. Malden, MA, Blackwell Futura Publishing, 2007.

30. Chakrabarti S, Morgan GJ, Kenny D, Walsh KP, Oslizlok P, Martin RP, Turner MS, et al. Initial experience of pacing with a lumenless lead system in patients with congenital heart disease. Pacing Clin Electrophysiol 2009; 32:1428-1433.

31. Sharma PS, Dandamudi G, Naperkowski A, Oren JW, Storm RH, Ellenbogen KA, Vijayaraman P. Permanent His-bundle pacing is feasible, safe, and superior to right ventricular pacing in routine clinical practice. Heart Rhythm 2015; 12:305-312.

32. Daccarett M, Segerson NM, Bradley DJ, Etheridge SP, Freedman RA, Saarel EV. Bipolar lumenless lead performance in children and adults with congenital heart disease. Congenital Heart Dis 2010; 5:149-156. 